\title{
Análisis multitemporal y predictivo del cambio de uso del suelo y pérdida de cobertura vegetal
}

Multitemporal and predictive analysis of the change in land use and loss of vegetation cover

\author{
Dante Leonardo Zumaeta Lozano \\ Bachiller en Ingeniería Ambiental, UNTRM-A
}

\section{RESUMEN}

El objetivo del presente estudio fue realizar un análisis multitemporal y predictivo del cambio de uso del suelo y pérdida de cobertura vegetal, utilizando técnicas de teledetección mediante un análisis geoespacial, aplicadas a imágenes satelitales LandSat. El estudio se realizó en la Microcuenca Yuyac, provincia de San Isidro de Maino y Magdalena, Chachapoyas, Amazonas; donde se evaluó el Cambio de uso del suelo y la pérdida de cobertura vegetal durante el periodo de 1998 al 2018, asimismo se realizó una simulación del escenario futuro al año 2030. En la microcuenca Yuyac se caracterizó seis clases de cobertura y usos del suelo (CUS). Se encontró que la cobertura boscosa (BO) sufrió una pérdida de 592.40 hectáreas durante el periodo de 20 años (1998 - 2018). De tal manera, en la simulación hacia el año 2030 se registró una pérdida de 129.40 hectáreas con una concentración extremadamente alta de $0.53 \%$. Se concluye que la degradación antrópica que influyó en el cambio de uso del suelo y pérdida de cobertura vegetal siendo las principales actividades la ganadería y agricultura, teniendo como resultado mayores espacios ocupados por pastos y cultivos que fueron incrementando con el paso del tiempo.

Palabras claves: Uso del suelo, cobertura vegetal

\begin{abstract}
The objective of this study was to carry out a multitemporal and predictive analysis of land use change and loss of vegetation cover, using remote sensing techniques through geospatial analysis, applied to LandSat satellite images. The study was carried out in the Yuyac Micro-basin, province of San Isidro de Maino and Magdalena, Chachapoyas, Amazonas; Where the change in land use and the loss of vegetation cover were evaluated during the period from 1998 to 2018, a simulation of the future scenario was also carried out to the year 2030. In the Yuyac micro-basin, six classes of cover and land uses were characterized ( CUS). It was found that the forest cover (BO) suffered a loss of 592.40 hectares during the 20-year period (1998 - 2018). In this way, in the simulation towards the year 2030 a loss of 129.40 hectares was registered with an extremely high concentration of $0.53 \%$. It is concluded that the anthropic degradation that influenced the change in land use and loss of plant cover, the main activities being livestock and agriculture, resulting in greater spaces occupied by pastures and crops that were increasing over time.
\end{abstract}

Key words: Land use, vegetation cover 


\section{INTRODUCCIÓN}

Los estudios sobre el cambio de uso del suelo y cobertura vegetal proporcionan la base para conocer las tendencias de los procesos de deforestación, degradación, desertificación y pérdida de la biodiversidad de una determinada área (García et al., 2012). Los modelos de cambio de uso de suelo y vegetación han sido desarrollados para determinar dónde, cómo y por qué ocurren estos cambios (Oliva, 2011), siendo importante dentro del estudio del Ordenamiento Ecológico Territorial, ya que permite visualizar los impactos pasados y presentes de los distintas actividades humanas en los usos del suelo y realizar una prospección tendencial que permita orientar en la búsqueda de estrategias para regular dichos impactos y tener un manejo más adecuado del territorio y de sus recursos naturales (Lambin et al., 2001).

Los ecosistemas terrestres han sufrido grandes cambios, la mayoría debido a la conversión de la cobertura del terreno a la degradación e intensificación del uso del suelo (Bocco et al., 2001). Son los cambios de uso y cobertura del suelo los que representan las evidencias más claras de intervención humana sobre el medio ambiente $y$ las modificaciones sobre el ecosistema terrestre (Britos y Barchuk, 2008 y Falcón, 2014). De este modo los cambios históricos en la cobertura y el uso de la tierra, en conjunto, ayudan a explicar la dinámica territorial; los cuales están asociados a factores biofísicos (suelo, vegetación, clima, topografía) y a factores humanos, cuyo estudio, constituye un insumo para diseñar acciones inherentes al Ordenamiento Territorial (Falcón, 2014).

Numerosos estudios señalan que el monitoreo y análisis de procesos como la deforestación, expansión urbana y sus consecuentes conversiones de cobertura naturales $y$ seminaturales se realiza mediante el estudio de los cambios de uso y cobertura del suelo (López et al., 2001; Azócar et al., 2006). Sin embargo, la literatura revisada indica, que no basta el monitoreo y análisis retrospectivo de los cambios de uso y cobertura del suelo, sino que es también necesaria la predicción a mediano y largo plazo de la transformación del territorio y sus efectos mediante la aplicación de modelos de simulación de cambios de uso y cobertura del suelo. Estos modelos juegan un papel importante al momento de explorar posibles desarrollos futuros, se podría explorar la incógnita de "qué pasaría si", a través de diferentes escenarios (Henríque et al., 2006; Verburg et al., 2006). La simulación de escenario de uso del suelo puede entonces ser tomada como una herramienta o un instrumento importante sobre el cual apoyarse para diseñar ordenaciones futuras del territorio (Gallardo, 2013).

Existen diversas investigaciones sobre cambio de uso del suelo realizados para periodos de análisis que abarcan los últimos 30 años con imágenes de satélite y también para periodos mayores, las cuales no solo han identificado los cambios comunes en la vegetación y el uso del suelo, sino que también han estudiado las causas de dichos cambios (Bhattarai et al., 2009). En cuanto a investigaciones sobre los modelos de cambios de usos del suelo y de simulaciones de escenarios, son muy numerosos, abarcando temas de deforestación en relación con la expansión de la agricultura o de los pastizales (Kok et al., 2001; Sangermano et al., 2012), el abandono de las tierras agrícolas (Rounsevell et al., 2003) así como el crecimiento y la dispersión urbana (Conway, 2009; Plata Rocha et al., 2010; Jokar et al., 2013).

Los antecedentes mencionados sobre cambios de cobertura y uso de suelo y la importancia de la simulación de escenarios futuros, han permitido sentar las bases para el desarrollo de esta investigación, teniendo en cuenta de las implicancias que representa sobre el medio ambiente y la sostenibilidad de las generaciones futuras. 


\section{MATERIAL Y MÉTODO}

\section{Área de estudio}

La Microcuenca Yuyac está ubicada en el distrito San Isidro de Maino y Magdalena, provincia de Chachapoyas, departamento de Amazonas. Abarca un área de 8034.1 hectáreas y un perímetro de 48.7 kilómetros (Figura 1). Ésta Microcuenca se encuentra ubicada a 1865 y 2786 m.s.n.m y su posición geográfica está determinada por las coordenadas $6^{\circ} 23^{\prime} 33.121^{\prime \prime} \mathrm{S}$ $77^{\circ} 49^{\prime} 2.812^{\prime \prime} \mathrm{W}$.

Cabe mencionar que la microcuenca Yuyac se encuentra inmersa en la unidad hidrográfica del Utcubamba.

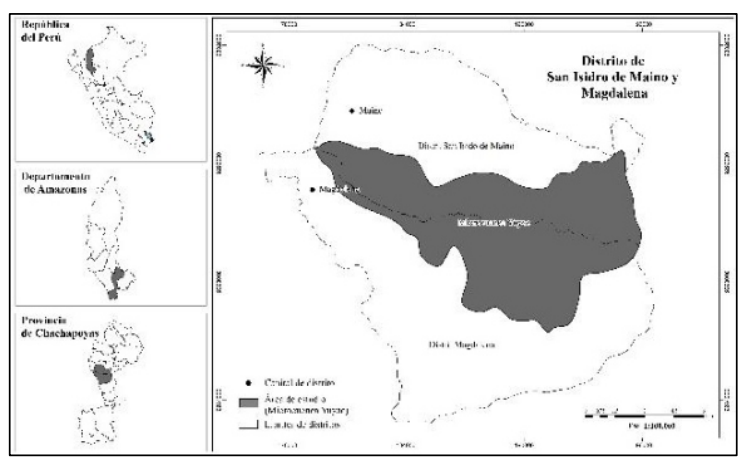

Figura 1. Mapa de ubicación del área de estudio (Microcuenca Yuyac)

\section{Metodología}

Se aplicó la herramienta CLC (Corine Land Cover) adaptada para Perú, con la finalidad de clasificar y analizar los cambios de uso del suelo y pérdida de cobertura vegetal (cobertura de bosque, cambios por pérdida natural, deforestación y degradación de bosques) de la Microcuenca Yuyac (MINAM, 2016a).

\section{Procesamiento y análisis de material satelital}

\section{- Delimitación del área de estudio}

Para la delimitación del área de estudio (Microcuenca Yuyac), se utilizó las curvas de nivel de la hoja 13h de la Carta Nacional del Instituto Geográfico Nacional (IGN) a escala 1:100 000, donde se manejó el programa ArcGis (v. 10.5.0.6491).

\section{Selección y adquisición de material satelital}

Las imágenes satelitales (1998, 2008 y 2018) fueron seleccionadas, tomando en cuenta criterios de calidad en el sentido de que las coberturas de nubes de las mismas no cubran la superficie del área de estudio y no tenga mayor al $10 \%$ de nubosidad, que las imágenes fueran aproximadamente tomadas en la misma época del año y que no presenten errores ni distorsiones. Para la adquisición de las imágenes satelitales se consultó y descargó mediante el catálogo del Instituto Nacional de Investigación Espacial (INPE) de Brasil y el portal de Servicio Geológico de los Estados Unidos (USGS, por sus siglas en inglés).

\section{- Tratamiento previo de las imágenes satelitales}

Para corregir aquellos errores y distorsiones que presenta las imágenes satelitales, se aplicó la corrección atmosférica y geométrica usando el programa QGIS 3.4 mediante el complemento Semi-Automatic Classification Plugin (SCP) y programa ArcGis (v. 10.5.0.6491). Para realizar la corrección geométrica (Georreferenciación) se tuvo en cuenta los puntos de control terrestre (GCP) levantados en campo (Cabrera et al., 2011).

Cuantificación del porcentaje de cambio de cobertura vegetal y determinación del grado de deforestación

Para realizar el análisis y cuantificación de los cambios en el uso del suelo se tomó en cuenta la leyenda Corine Land Cover adaptada para Perú (MINAM, 2016a), donde se identificó seis Clases de Uso del Suelo (CUS) mediante salidas a campo.

Clases de cobertura y uso del suelo identificadas en campo e imágenes satelitales de alta resolución: Áreas artificializadas (AA), Mosaico de pastos y cultivos (MPC), Bosques (BO), Herbazal / Pajonal (HP), Arbustal (AR) y Vegetación arbustiva / Herbácea (VAH). 


\section{- Clasificación supervisada de máxima probabilidad}

Este proceso se realizó mediante la identificación de las firmas espectrales levantadas en campo (Rawat y Kumar, 2015). Se realizó la clasificación supervisada de cada imagen satelital (1998, 2008 y 2018) para atribuir categorías predeterminadas de los tipos de cobertura (uso del suelo) del área de estudio.

\section{- Interpretación visual interdependiente}

Las imágenes fueron interpretadas visualmente utilizando el método de interpretación interdependiente de la FAO (2001). Se interpretó los polígonos de la primera fecha (2018) para luego ser empleados los mismos polígonos en las fechas inferiores (1998 y 2008), donde solo se modificó los segmentos que generaron un cambio.

\section{- Limpieza y corrección topológica}

Se tuvo en cuenta la escala de trabajo, donde se eliminó los polígonos con áreas menores a 1.5 hectáreas, según la tabla de Área Mínima Cartografiable (Vargas, 1992). Se realizó la corrección topológica mediante la fusión de los polígonos adyacentes que tenía el área más grande.

\section{- Evaluación de exactitud temática}

Para validad la precisión se tuvo en cuenta los 196 puntos de verificación obtenidos de acuerdo a la formula señalada por Chuvieco (1998). Posterior a ello se elaboró la matriz de confusión (Chuvieco, 2002) sobre el cual se hizo el cálculo de métricas de precisión con la finalidad de evaluar la exactitud a nivel de clases.

También se hizo el cálculo del Índice de Kappa (k) para medir la fuerza de concordancia para saber si el estudio realizado se encuentra en un rango óptimo (Congalton y Green, 2009; López y Fernández, 1999).

\section{- Tasa de cambio y matrices de transición}

El cálculo de la tasa de cambio se realizó mediante la ecuación planteada por la FAO (1996).

$$
S=\left(\frac{S_{2}}{S_{1}}\right)^{\frac{1}{\left(t_{2}-t_{1}\right)}}-1
$$

Donde, $S_{1}$ y $S_{2}$ son las superficies de CUS en la fecha $t_{1}$ y $t_{2}$.

Posterior a ellos se elaboró mapas de cambios de cobertura y uso del suelo para ambos periodos, matrices de tabulación cruzada y mapa de deforestación acumulada. Estas matrices se usaron para distinguir las transiciones de las diferentes clases evaluadas, detectar los cambios y hacer un análisis de los patrones reales que conllevan a cambios (Pontius et al., 2004).

\section{Determinación de la concentración de la pérdida de cobertura vegetal natural}

Se analizó la información de pérdida de cobertura vegetal natural (cobertura boscosa), para los períodos 1998 - 2008 y 2008 - 2018, con la finalidad de determinar si la pérdida está concentrada en ciertas áreas dentro de ámbito de la Microcuenca y cuánto representa esta concentración con respecto al área total, para ello se utilizó la metodología de análisis de Densidad Kernel, tomando como referencia un análisis similar del MINAM (2016b).

\section{Estimación de escenario futuro al año 2030}

Para realizar la predicción y validación del cambio de uso del suelo y pérdida de cobertura vegetal al 2030, se utilizó el complemento MOLUSCE del programa QGIS (v. 2.18.27) (Gómez, 2017). Se tuvo en cuenta las clases de cobertura y usos de los suelos (CUS) generados de periodos anteriores (1998, 2008 y 2018) (Soares-Filho et al., 2002).

Se trabajó sobre 2 mapas de CUS y variables espaciales de predicción (distancia a carreteras, centros poblados, red hídrica, pendiente $\mathrm{y}$ altitud) correspondientes al tiempo inicial $\mathrm{T} 1 \mathrm{y}$ tiempo final T2. Para validar los resultados se 
Tabla 2. Áreas de cambio de cobertura y uso del suelo (CUS) por cada periodo.

\begin{tabular}{|l|c|c|c|}
\hline \multirow{2}{*}{ Transición de CUS } & \multirow{2}{*}{ Símbolo } & \multicolumn{2}{c|}{ Área (ha) } \\
\cline { 3 - 4 } & & $\mathbf{1 9 9 8 - \mathbf { 2 0 0 8 }}$ & $\mathbf{2 0 0 8} \mathbf{- 2 0 1 8}$ \\
\hline Cambio a CUS antrópico & & 351.0 & 170.2 \\
\hline Permanencia de CUS antrópico & & 1331.3 & 1682.3 \\
\hline Permanencia de CUS natural & & 6351.8 & 6181.6 \\
\hline \multicolumn{2}{|c|}{ TOTAL } & $\mathbf{8 0 3 4 . 1}$ & $\mathbf{8 0 3 4 . 1}$ \\
\hline
\end{tabular}

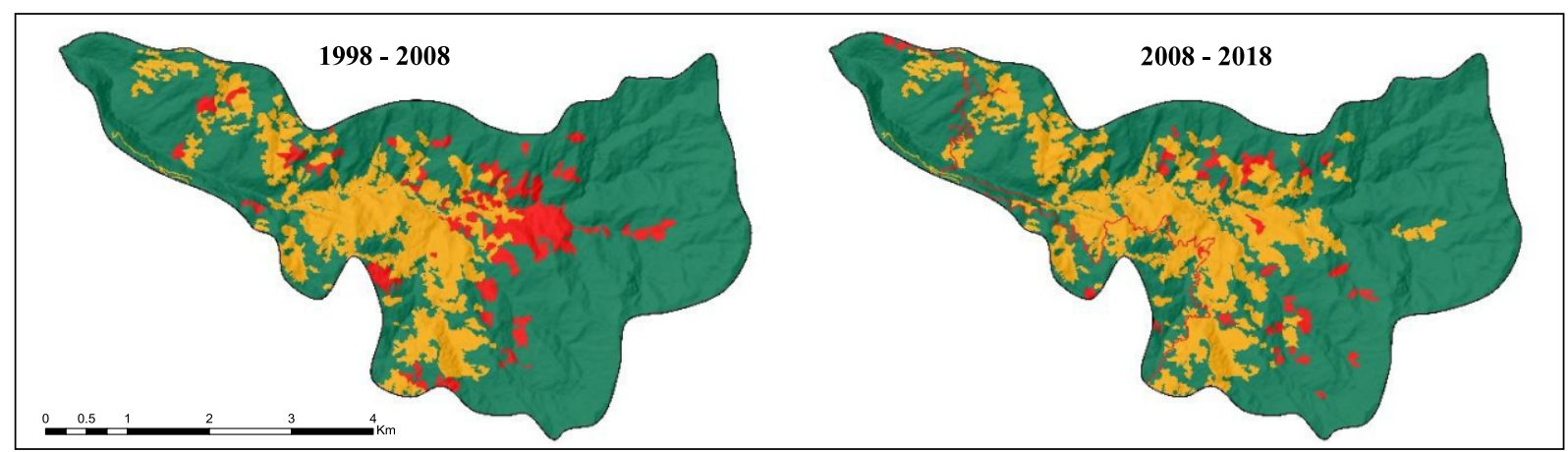

Figura 5. Mapas de la dinámica de cambio de cobertura y uso del suelo (CCUS) por cada periodo de la Microcuenca Yuyac.

Concentración de pérdida de cobertura vegetal natural en la Microcuenca Yuyac

Durante los dos periodos analizados (19982008 y 2008-2018) se puede observar que la concentración de pérdida de cobertura boscosa va desde baja a extremadamente alta; sin embargo en el primero periodo, la tasa de pérdida es superior respecto al segundo periodo, teniendo una concentración de pérdida extremadamente alta de 105.6 ha $(1.31 \%$ del área total).

Tabla 3. Áreas por concentración y densidad de la pérdida de cobertura vegetal en la Microcuenca Yuyac.

\begin{tabular}{|c|c|c|c|c|c|}
\hline \multirow{2}{*}{$\begin{array}{c}\text { Concentración de la } \\
\text { pérdida de cobertura }\end{array}$} & \multirow{2}{*}{ Símbolo } & \multicolumn{2}{|c|}{$1998-\mathbf{2 0 0 8}$} & \multicolumn{2}{c|}{$\mathbf{2 0 0 8 - 2 0 1 8}$} \\
\cline { 3 - 6 } & \multicolumn{2}{|c|}{ Área } & \multicolumn{2}{c|}{ Área } \\
\cline { 3 - 5 } & ha & \% & Ha & \% \\
\hline Baja & 6445.5 & 80.23 & 6791.8 & 84.54 \\
\hline Media & 736.7 & 9.17 & 682.5 & 8.50 \\
\hline Alta & 502.8 & 6.26 & 356.6 & 4.44 \\
\hline Muy alta & 243.5 & 3.03 & 146.9 & 1.83 \\
\hline Extremadamente alta & 105.6 & 1.31 & 56.3 & 0.70 \\
\hline Total & $\mathbf{8 0 3 4 . 1}$ & $\mathbf{1 0 0 . 0 0}$ & $\mathbf{8 0 3 4 . 1}$ & $\mathbf{1 0 0 . 0 0}$ \\
\hline
\end{tabular}
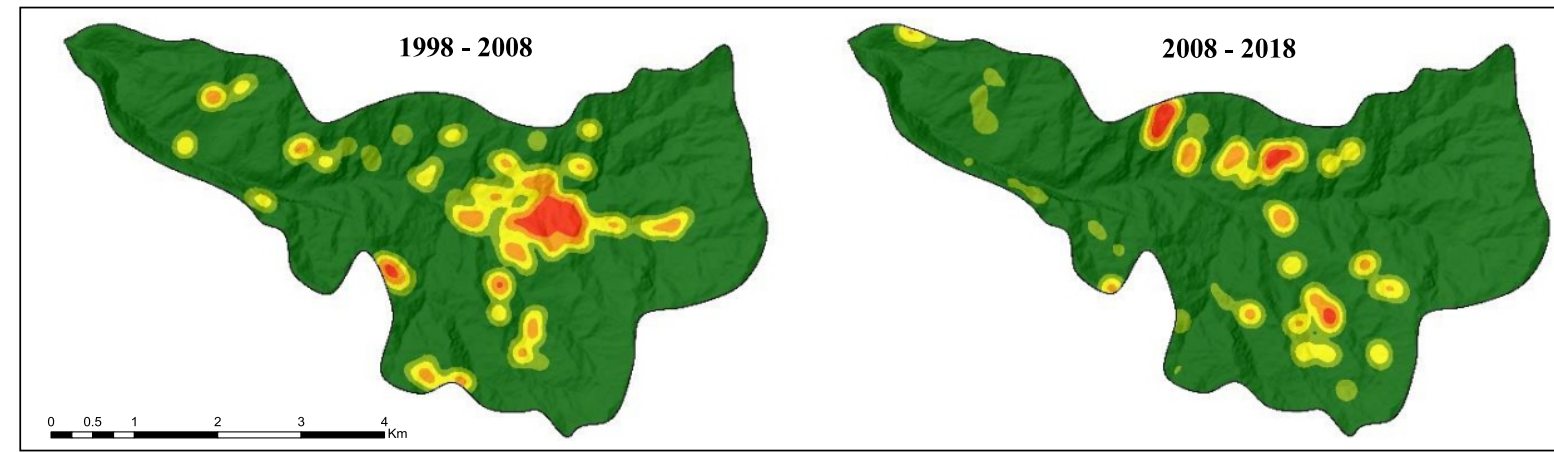

Figura 6. Mapa de concentración de la pérdida de cobertura vegetal de la Microcuenca Yuyac. 


\section{Escenario de la Microcuenca Yuyac al 2030}

La cobertura y uso del suelo de mayor predominancia hacia el año 2030 está conformada por la cobertura Boscosa (BO) y Mosaico de pastos y cultivos (MPC) con áreas de 3252.5 ha ( $40.5 \%$ del área total) y 1934.0 ha (24.1\% del área total), respectivamente.

Tabla 4. Área de cobertura y uso del suelo hacia el año 2030.

\begin{tabular}{|c|c|c|}
\hline Clasificación & Símbolo & Área (ha) \\
\hline BO & & 3252.5 \\
\hline MPC & & 1934.0 \\
\hline HP & & 905.1 \\
\hline AA & 32.3 \\
\hline AR & 1431.3 \\
\hline VAH & 478.9 \\
\hline \multicolumn{2}{|c|}{ TOTAL } & $\mathbf{8 0 3 4 . 1}$ \\
\hline
\end{tabular}

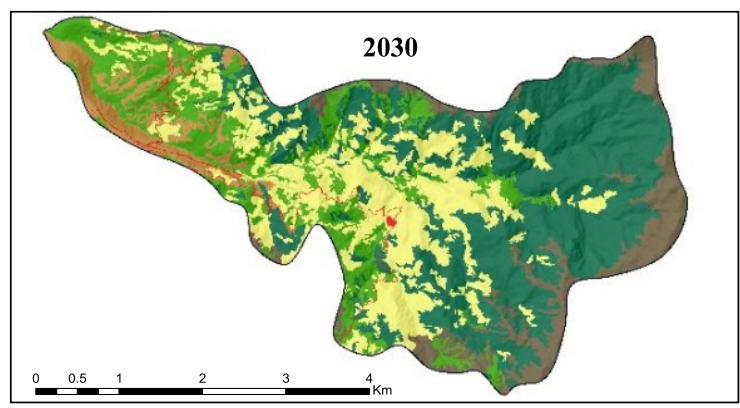

Figura 7. Mapa de cobertura y uso del suelo (CUS) de la Microcuenca Yuyac - 2030.

La probabilidad de cambio de la cobertura y uso del suelo hacia el año 2030 los resultados del escenario indican que se perderá alrededor de 129.40 ha de cobertura boscosa (BO), las cuales pasarían con mayor cantidad a áreas de uso antrópico (MPC).

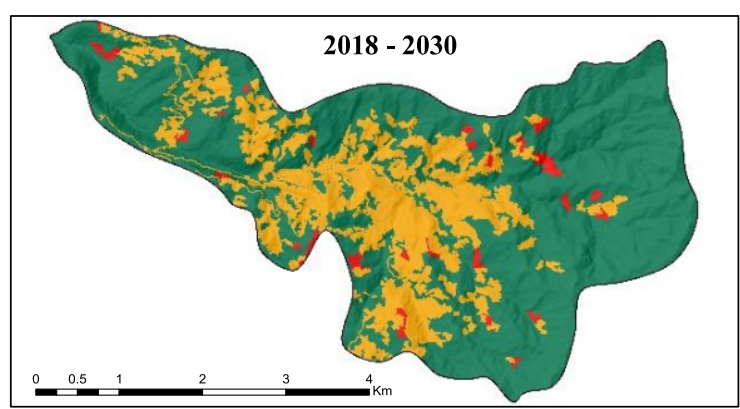

Figura 8. Mapa de la dinámica de cambio de cobertura y uso del suelo (CCUS) de la Microcuenca Yuyac, periodo 2018 - 2030.
La distribución de pérdida de cobertura boscosa durante el periodo 2018 - 2030 va desde baja a extremadamente alta. Se puede apreciar que, la probabilidad que ocurra una concentración de pérdida extremadamente alta en el área de estudio es de 42.3 ha.

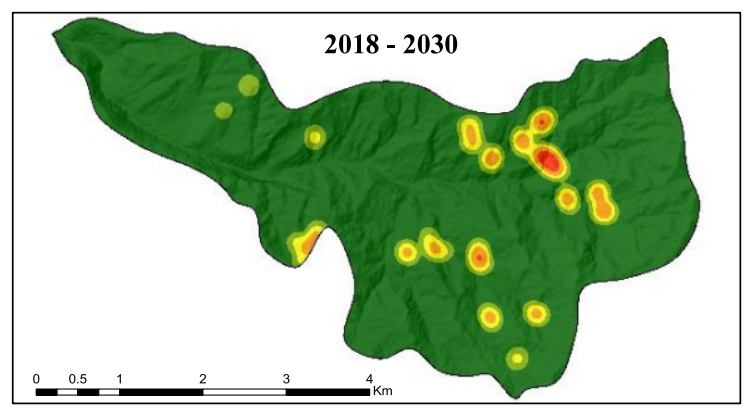

Figura 9. Mapa de concentración de la pérdida de cobertura vegetal de la Microcuenca Yuyac, periodo 2018 - 2030.

\section{Variable del cambio de uso del suelo $y$ pérdida de cobertura vegetal}

Las variables de mayor importancia que intervinieron en dichos cambios están relacionadas principalmente con las actividades de ganadería y agricultura, teniendo como resultado mayores espacios ocupados por pastos y cultivos que fueron incrementándose con el paso del tiempo, reduciendo la cobertura vegetal natural. Otra de las variables que influyó en menor medida se encuentra asociada con el proceso normal de la expansión de áreas urbanas, reflejado en la ampliación de infraestructura como vías de acceso (carreteras, puentes) y viviendas.

\section{DISCUSIóN}

La tasa de deforestación y cambios de cobertura vegetal varían de un lugar a otro, y están en función de las actividades que desarrolla el hombre y de la magnitud en las que la realiza, sin embargo, para Rico (2016) la ganadería basada en pastoreo o también denominada ganadería extensiva es la que realiza cambios considerables en los paisajes rurales y debe reconocerse como un proceso de enormes repercusiones ambientales y sociales. 
La dinámica de cambio que experimentaron las distintas coberturas que comprende la Microcuenca en estudio, según CamachoSanabria (2017) están relacionadas con el proceso de conversión de sistemas agrícolas, puesto que para el periodo 1998-2008, todas las categorías sufrieron pérdidas y ganancias, a diferencia de cobertura $\mathrm{BO}$ que sólo registró pérdidas y AA que por su parte solo tuvo ganancias, siendo la cobertura $\mathrm{BO}$ la que mayor pérdida registró con 474.20 ha y cuyos espacios fueron utilizados para establecer principalmente zonas agropecuarias, incrementando el área de MPC, paralelo a ello las zonas urbanas se expandieron, incrementándose la categoría de AA cuyos espacios procedieron de la pérdida o transformación de la categoría MPC, mostrando cambios característicos de los sistemas agrícolas.

En cuanto a la probabilidad del cambio de uso del suelo y pérdida de cobertura vegetal hacia el año 2030 en la Microcuenca Yuyac; los resultados del escenario indican que, si la tendencia actual continúa para el año 2030 se perderán alrededor de 129.40 ha de cobertura boscosa (BO), las cuales pasarían en mayor proporción a áreas de uso antrópico (MPC) siendo levemente superior a la perdida ocurrida en el periodo 2008-2018. Estos resultados tienen similitud en el pronóstico de deforestación con otros estudios realizados a nivel internacional como el de Ortega (2018) que arrojó resultados para el Municipio de Villagarzon, Colombia pronosticando una reducción del 11\% (14 749 ha) de cobertura natural en un periodo de 30 años, con una tasa de deforestación de 509 ha/año, del mismo modo en el Cantón Cuenca, Ecuador, la simulación del modelo al año 2010 y 2030, presenta la tendencia de disminución de cobertura vegetal (páramo, vegetación herbácea $\mathrm{y}$ leñosa) generando aumento de pastos y cultivos (Pinos, 2016).

\section{CONCLUSIONES}

Se logró caracterizar seis clases de cobertura y uso del suelo (Áreas artificializadas - AA, Mosaico de pastos y cultivos - MPC, Bosques -
BO, Herbazal / Pajonal - HP, Arbustal - AR y Vegetación arbustiva / Herbácea - VAH) de las cuales la cobertura boscosa es la que mayor superficie abarca, seguida de la cobertura de mosaico de pastos y cultivos. Se determinó que la cobertura boscosa registró mayores pérdidas en los diferentes periodos, registrando una pérdida de 474.20 hectáreas para el periodo 1998 - 2008 y 118.20 hectáreas para el periodo $2008-2018$.

La simulación de los cambios de uso suelo y pérdida de cobertura vegetal al año 2030 de la Microcuenca Yuyac registró la disminución de cobertura boscosa (BO) con una pérdida de 129.40 hectáreas y el crecimiento de la clase de mosaico de pastos y cultivos (MPC) con una ganancia de 161.90 hectáreas del año 2018 al 2030.

De acuerdo al análisis de mapas, consulta en base de datos referente al área de estudio y fuentes bibliográficas, se logró identificar que las variables de mayor importancia relacionadas con los cambios de uso del suelo y pérdida de cobertura vegetal están dadas por la naturaleza agropecuaria de la zona (ganadería y agricultura) y la dinámica normal de la población reflejada en la expansión urbana y ampliación de infraestructura como vías de acceso (carreteras y puente).

\section{REFERENCIAS BIBLIOGRÁFICAS}

Azócar, G., Sanhueza, R. y Henriquez, C. (2006). Cambio en los patrones de crecimiento en una ciudad intermedia: el caso de Chillán en Chile Central. Revista EURE 21 (87): 79-92.

Bhattarai, K., Conway, D., y Yousef, M. (2009). Determinants of deforestation in Nepal's Central Development Region. Journal of Environmental Management, 91: 471-488.

Britos, A.H., y Barchuk, A.H. (2008). Cambios en la cobertura y en el uso de la tierra en dos sitios del Chaco Árido del noroeste de Córdoba, Argentina. Agriscienta, 15(2): 97-110. 
Bocco, G., Mendoza, M., y Masera, O. (2001). La dinámica del cambio de uso de suelo en Michoacan. Una propuesta metodológica para el estudio de los procesos de deforestación. México: Instituto de Geografía-UNAM.

Cabrera, E., Galindo, G., y Vargas, D.M. (2011). Protocolo de Procesamiento Digital de Imágenes para la Cuantificación de la Deforestación en Colombia, Nivel Nacional Escalas Gruesa y Escala Fina. Bogotá D.C., Colombia: Instituto de Hidrología, Meteorología, y Estudios Ambientales-IDEAM.

Camacho-Sanabria, R., Camacho-Sanabria, J., Balderas-Plata, M., y Sánchez-López, M. (2017). Cambios de cobertura y uso de suelo: Estudio de caso en Progreso Hidalgo, estado de México. Madera y Bosques, 23(3):39-60.

Chuvieco, E. (1998). El factor temporal en teledetección: evolución fenomenológica y análisis de cambios. Revista de teledetección, 10, 1-9.

Chuvieco, E. (2002). Teledetección ambiental: la observación de la Tierra desde el Espacio. Barcelona, España: Ariel Ciencia.

Conway, T.M. (2009). The impact of class resolution in land use change models. Computers, Environment and Urban Systems, 33, 269-277.

Congalton, R. y Green, K. (2009). Assessing the Accuracy of Remotely Sensed Data: Principles and Practices. Boca Raton, FL: CRC Press.

Falcón, O. (2014). Dinámica de cambio en la cobertura/uso de suelo, en una región del Estado de Quintana Roo, México: El impacto de las políticas gubernamentales sobre el manejo forestal. Tesis de Maestría, Universidad Nacional Autónoma de México.

Gallardo, M. (2013). Cambios de uso del suelo $y$ simulaciones de escenarios en la comunidad de Madrid. Tesis doctoral. Universidad Complutense de Madrid, España.
García, J., Cedillo, J., Juan, J., y Balderas, M. (2012). Proceso de cambio en el uso de suelo de una Microcuenca en el altiplano Mexicano. El caso del río San José en el estado de México. Papeles de Geografia, (55):63-73.

Gómez, L. N. (2017). Inteligencia artificial aplicada al territorio. Universidad Distrital Francisco José de Caldas. Recuperado de: https://intelaplite.wordpress.com/2017/08/ 28/molusce-de-qgis/

Ibáñez, N., y Damman, G. (2014). Cambios de cobertura de los usos de suelo para la elaboración de escenarios territoriales en la región Apurimac. Zonas Áridas 15(1), 4867.

Jokar, J., Helbich, M., Kainz, W., y Darvishi Boloorani, A. (2013). Integration of logistic regression, Markov chain and cellular automata models to simulate urban expansion. International Journal of Applied Earth Observation and Geoinformation, 21, 265-275.

Kok, K., Farrow, A., Veldkamp, A., y Verburg, P.H. (2001): A method and application of multi-scale validation in spatial land use models. Agriculture, Ecosystems and Environment, 85, 223-238.

Lambin, E., Tuner, L., Geist, H., Agbola, S., Angelsen, A., Bruce, J., Coomes, O., Dirzo, R., Fischer, G., y Folke, C. (2001). Las causas del uso de la tierra y el cambio de la cobertura terrestre: ir más allá de los mitos. Global Environmental Change,, 11, 261-269.

López, E., Bocco, G. y Mendoza, M. (2001). Predicción del cambio de cobertura y uso del suelo. El caso de la ciudad de Morelia. Investigaciones Geográficas. Boletín del Instituto de Geografia UNAM México, 45: 56-76.

López de Ullibarri Galparsoro I, Pita Fernández, S., 1999, Medición de concordancia: Indice de Kappa, Unidad de Epidemiologia Clínica y Bioestadística. Complexo Hospitalario-Universitario Juan Canalejo. A Coruña (España). 
MINAM. (2016a). R.M. $\quad \mathrm{N}^{\circ}$ 081-2016MINAM: Procedimiento técnico $y$ metodológico para la elaboración del estudio especializado de análisis de los cambios de la cobertura y uso de la tierra. Lima, Perú: MINAM.

MINAM. (2016b). Mapas kernel como indicador de la concentración de la pérdida de bosques húmedos amazónicos del Perú. Lima, Perú: MINAM.

Oliva, P. (2011). Análisis de cambio de uso de suelo mediante percepción remota en el municipio de Valle de Santiago. Tesis para obtener el grado de Maestro en Geomática. Centro de Investigación en Geografía y Geomática, México D. F.

Ortega, D. A. (2018). Transformación del paisaje natural del Municipio de Villagarzón, Colombia, periodo 1985 a 2014, su proyección a futuro 2044 y ganadería actual. Tesis de maestría. Universidad de Salzburg, Departamento de Geomática, Colombia.

Pinos, N. J. (2016). Prospectiva del uso del suelo y cobertura vegetal en el ordenamiento territorial. Estoa 9(5): 7-19.

Plata, W., Gómez, M., y Bosque, J. (2010). Desarrollo de modelos de crecimiento urbano óptimo para la Comunidad de Madrid aplicando métodos de evaluación multicriterio y Sistemas de Información Geográfica. Geofocus, 10, 103-134.

Pontius, R. G., Shusas, E. y Mceachern, M. (2004). Detecting important categorical land changes while accounting for persistence. Agriculture, Ecosystems and Environment, 101(2-3), 251-268.

Rawat, J. S. y Kumar, M. (2015). Monitoring land use/cover change using remote sensing and GIS techniques: A case study of Hawalbagh block, district Almora, Uttarakhand, India. The Egyptian Journal of Remote Sensing and Space Science, 18(1), 77-84.

Rico, G. (2016). Colombia: La ganadería extensiva está acabando con los bosques. Colombia: Mongaby.
Rounsevell, M.D., Annetts, J.E., Audsley, E., Mayr, T., y Reginster, I. (2003). Modelling the spatial distribution of agricultural land use at the regional scale. Agriculture, Ecosystems \& Environment, 95: 465-479.

Sangermano, F., Toledano, J., y Eastman, J.R. (2012). Land cover change in the Bolivian Amazon and its implications for REDD+ and endemic biodiversity. Landscape Ecology, 27, 871-584.

SEMARNAT (Secretaria de Medio Ambiente y Recursos Naturales) (2002). Informe de la situación de medio ambiente en México, Compendio de estadísticas ambientales. México: Dirección General de Estadística e información ambiental.

Soares-Filho, B., Coutinho, G., y Lopes, C. (2002). DINAMICA-a stochastic cellular autómata model designed to simulate the landscape dynamics in an Amazonian colonization frontier. Ecological Modelling 154, 217-235.

UNIBA (Centro Universitario Internacional de Barcelona) (2018). Analizando las dinámicas de cambios de uso de suelo. España, Barcelona: Universidad de Barcelona.

Vargas G., E. (1992). Análisis y clasificación del uso y cobertura de la tierra con interpretación de imágenes. Colombia: IGAC (Instituto Geográfico Agustín Codazzi).

Verburg, P.H., Kok, K., Pontius, R. G., y Veldkamp, A. (2006). Modeling land-use and land-cover change. In Land-use and land-cover change. Local proccesses and Global Impacts. Berlin: E.F. Lambin and H.J. Geist. 